\title{
Applications of nanotechnology in biomedicine
}

\author{
WU JiMin \& LI ZiJian* \\ Institute of Vascular Medicine, Peking University Third Hospital, Key Laboratory of Cardiovascular Molecular Biology and Regulatory Peptides, \\ Ministry of Health, Key Laboratory of Molecular Cardiovascular Sciences, Ministry of Education and Beijing Key Laboratory of Cardiovascular \\ Receptors Research, Beijing 100191, China
}

Received May 7, 2013; accepted July 24, 2013; published online August 22, 2013

Citation: Wu J M, Li Z J. Applications of nanotechnology in biomedicine. Chin Sci Bull, 2013, 58: 4515-4518, doi: 10.1007/s11434-013-6063-0

Nanomedicine is the application of nanotechnology in treatment, diagnosis, monitoring and control of biological systems, and is at the leading-edge of clinical medicine and preclinical research. Increasing attention has been paid to the application of nanotechnology in medicine recently (Figure 1). Nanotechnology means the control of matter and processes at a nanoscale $(1-100 \mathrm{~nm})$ in one or more dimensions [1]. The material and devices operated at the nanoscale usually have different physical properties compared with those at the normal size. Nanomedicine-based approaches have thus an unprecedented potential to better control biological processes and to improve the detection, therapy and prevention of multiple diseases. The applications of nanotechnology and its safety have become the highlight of current biomedical research. This paper focuses on the main nanotechnologies and their biosafety encountered in biomedical research, diagnosis and therapy (Figure 2).

\section{Nanotechnology in biomedical analysis and research}

Fluorescence imaging has been widely used in biology and medicine. Single-molecule detection (SMD) can detect signals from individual molecules, which removes the average effect in classical ensemble experiments [2,3]. Besides the confocal fluorescence microscopy, the main SMD techniques include total internal reflection fluorescence microscopy (TIRFM), single-molecule fluorescence resonance energy transfer (smFRET), cylindrical illumination confocal

*Corresponding author (email: lizijian@bjmu.edu.cn)

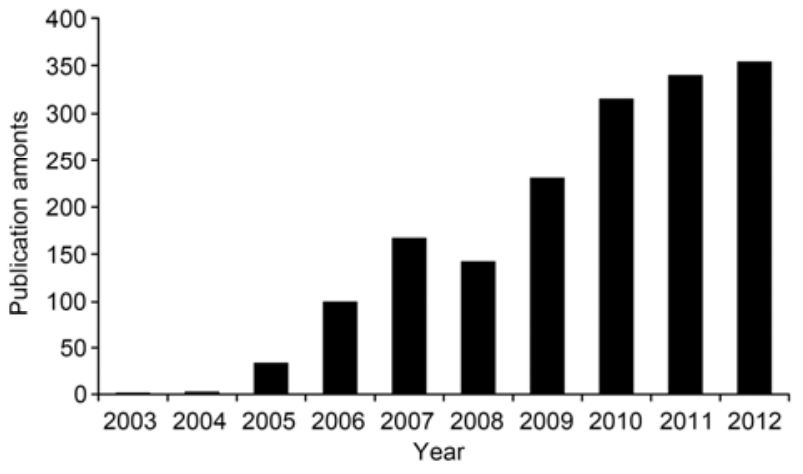

Figure 1 The publication amounts of nanomedicine related papers in PubMed in the last 10 years.

spectroscopy (CICS), epi-fluorescence microscopy, confocal microscopy, quasi-TIRFM and single-point edge excitation subdiffraction microscopy (SPEED). SMD techniques are widely used in membrane protein research. For instance, in the experiment regarding TGF-beta type II receptors of neonatal rat cardiomyocytes, the dimerized receptors were found to increase in hypertrophic cardiomyocytes by SMD [4], which infers the functioning of TGF-beta signaling in cardiac remodeling. Another study tracked the $\alpha_{1 \mathrm{~A}}$-adrenergic receptor, and found that endocytic pathway is involved in $\alpha_{1 \mathrm{~A}}$-AR-induced ERK1/2 activation, and is independent of $\mathrm{G}(\mathrm{q}) / \mathrm{PLC} / \mathrm{PKC}$ signaling [5]. Moreover, some nanomaterials are potential florescence probes in relevant technologies. For instance, quantum dots are of high photo stability, high identification accuracy and controllable colors, and have been used as fluorescence imaging probes, fluorescent protein FRET probes and molecular beacons [6]. 


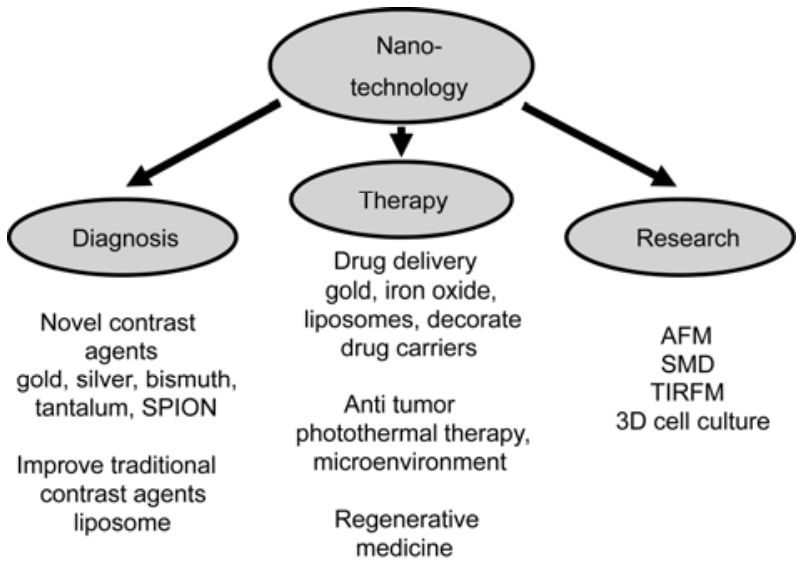

Figure 2 The nanotechnology in the biomedical research and the application of nanomaterial in the diagnosis and therapy of diseases.

Atomic force microscopy (AFM) is a powerful tool in the study of biomolecular interactions, and can measure the binding forces at the molecular level of protein/DNA, DNA/ DNA, ligand/receptor and other biomolecule pairs. It is based on the nanomechanical cantilever sensors and microcantilever-based biosensors, which can provide rapid and sensitive in situ detection [7]. AFM has been used to study the influences of substrate stiffness on mechanical properties of cardiac myocytes and fibroblasts [8]. Cardiac fibroblasts, but not myocytes, displayed the non-monotonic dependence on substrate stiffness with a maximum elastic modulus, which was further found to be regulated by actin filaments. The nanoscale architecture of microbes and the mechanic properties of viruses can also be detected by the AFM [9,10]. Moreover, The AFM is an optimal choice to determine the structure, conformational changes and dynamics of membrane proteins under near-physiological conditions, so as to investigate the cell communication, signal transduction, etc. [11].

\section{Nanotechnology in diagnosis}

Nanomaterials have been used in diagnosis mostly as contrast agent in molecular imaging. They are usually in small size and of much higher surface area to volume ratio, thus their surface can be decorated with more therapeutic molecules, imaging agents, targeting ligands, and nucleic acids. When used as contrast agents, they can circulate in the blood for longer time with higher sensitivity and possibly fewer side-effects [1]. X-ray computed tomography (CT) is widely used in diagnostic medicine. Many kinds of nanomaterials have shown great potentials as CT contrast agents, such as gold, silver, bismuth, and tantalum nanomaterials [6]. Gold nanoparticles have higher atomic number, X-ray absorption coefficient, and longer blood circulation half-life than clinically used CT contrast agents [6]. They are also utilized in light scattering imaging, two-photon fluorescence
(TPF) imaging and photoacoustic imaging in the cell imaging-based detection for tumors [12]. Besides, entrapment with Liposomes can prolong the circulation half-time of traditional contrast agents as iodinated ones [6].

Magnetic resonance imaging (MRI) provides superior contrast in soft tissue imaging and has no radiation. Compared with gadolinium-based MRI contrast agents, nanoparticle MRI contrast agents circulate longer in the blood, and have higher sensitivity and fewer side-effects [6]. Combining positron emission tomography (PET) with the highresolution, spectroscopic, and contrast enhancement abilities of MRI would make a breakthrough in the detection and monitoring of diseases. Superparamagnetic iron oxide nanoparticles (SPIONs) are the most promising nanoparticle system as MRI and PET contrast agents $[6,13]$. These nanocrystals containing thousands of $\mathrm{Fe}$ atoms can generate signal contrast with several orders of magnitude higher than a gadolinium chelate. A variety of SPIONs have been synthesized with differences in the formation of cores and surface coating, and many of them are currently under clinical trials $[14,15]$.

\section{Nanotechnology in therapy}

Nanomaterials have been introduced to the therapy of multiple diseases, including drug delivery system and nanodrugs. Drug delivery is one of the typical applications of nanomaterials in medicine. For example, tumor targeting, imaging and drug delivery can be accomplished by administrated gold nanoparticles and nanorods, iron oxide nanoworms and drug loaded liposomes [6,12]. Some other nanomaterials can be used to decorate gold nanoparticles to improve the capability [16]. The nanotechnology has also been applied to the intelligent drug-delivery systems [17] and implantable drug-delivery systems $[18,19]$, so as to realize the controlled and targeted release of therapeutic drugs. Besides drug delivery, nanomaterials have been adopted in some specific tumor therapies. Au nanoparticles have the potential to be developed as novel contrast agents in photothermal cancer therapy [20]. They concentrate in the diseased region, absorb light and convert it into heat to destroy the malignant cells. Gd@ $\mathrm{C}_{82}(\mathrm{OH})_{22}$ nanoparticles have been demonstrated to be a potent antitumor nanomedicine acting on the tumor microenvironment [1]. They have no direct tumor cytotoxicity, and their antineoplastic activity is based on the inhibition of oxidation stress and angiogenesis, the activation of immune reaction, the imprisoning of cancer cells, and the reversing of drug-resistance combination. Au nanoparticles have similar impact on tumor microenvironment [12].

The application of nanotechnology has opened a new realm in the advance of regenerative medicine. The development of nanotechnology offers more opportunities of applying stem cells in the regeneration of tissues and organs. 
Rebuilding tissue-like structures in vitro is important for both fundamental cell biology research and tissue engineering (TE). The top-down or bottom-up approach is adopted in TE $[21,22]$. The top-down approach is mainly seeding cells into the biomaterial scaffolds and forming similar macroscopic tissue features. The bottom-up approach focuses on the functional microscale features with physical and/or chemical methods. Microscale methods of the bottom-up approach allow cells to form three-dimensional microstructures, and recapitulate the extracellular microenvironment around the cells for engineering microtissue constructs. A variety of nanomaterials are employed in regenerative medicine. Iron oxides like inorganic nanoparticles are among the most promising candidates in stem-cell regeneration [22]. Titanium oxide $\left(\mathrm{TiO}_{2}\right)$ surface nanostructures were reported to be utilized in orthopedic implant [23]. $\mathrm{TiO}_{2}$ nanotube exerts important impacts on osteogenic cells, and is promising in the orthopedic material surface designs.

\section{The biosafety of nanotechnology}

Biosafety is mostly concerned in nanotechnological applications. It is important to better understanding the metabolic fate and biological effect in cells or organs as increasing nanomaterials are hopeful materials to be applied in medicine. The toxicity of most nanomaterials applied in biomedicine has been examined in preclinical research in that the low toxicity and optimal biocompatibility are necessary for their clinical applications. $\mathrm{Gd} @ \mathrm{C}_{82}(\mathrm{OH})_{22}$ nanoparticles have no discernible toxic effects either in vitro or in vivo [24]. Carbon nanotubes (CNTs) are nanomaterials widely applied in biomedicine. However, the high cytotoxicity limits their use in humans. Water-soluble single-walled carbon nanotubes and fullerene $\mathrm{C}_{70}\left(\mathrm{C}(\mathrm{COOH})_{2}\right)_{4-8}$ exerted more serious adverse effects on BY-2 cells [25]. Gold nanoparticles are considered to be relatively safe as elemental $\mathrm{Au}$ is highly inert. However, some study found it was hazardous in some cases [26]. However, those undesired side effects can be avoided by simply changing the capping agents. The functionalization of CNTs can improve their solubility and biocompatibility, and change the interaction with cells, resulting in reduced cytotoxicity [27].

The cell uptake and metabolic fate of nanomaterials were also studied. The cellular uptake of nanoparticles depended on the time of incubation and the concentration of nanoparticles in the medium $[28,29]$. Up-taken CNTs are shown to traffic through the different cellular barriers by energy-independent mechanisms [30]. SPIONs entered cells through multiple endocytic pathways and could be subsequently passaged to daughter cells, degraded in the lysosome, or exocytosed out of the cells [1]. In this process, SPIONs did not induce cell damage, except for the effect on iron metabolism. Better understanding of the nanomaterial metabolic pathways will contribute to the development of
Table 1 Typical applications of nanotechnology in biomedical detection and in diagnosis and therapy

\begin{tabular}{ll}
\hline \multicolumn{1}{c}{ Biomedical detection } & \multicolumn{1}{c}{ Diagnosis and therapy } \\
\hline Bio-detection of pathogens & Tissue engineering \\
Detection of proteins & Photothermal therapy \\
Detection of proteins & MRI and CT contrast agents \\
Probing of DNA structure & Contrast enhancement \\
Tissue engineering & Drug and gene delivery \\
Separation of biomolecules and cells & Vaccination applications \\
Fluorescent biological markers & Nanorobotics \\
Phagokinetic studies & \\
Artificial cells & \\
Biosensors & \\
\hline
\end{tabular}

nanobiology and advocate the secure use of nanotechonology in medicine.

Nanoparticle toxicity is routinely assessed in cultured cells in vitro. The data from these studies require verification from animal experiments, as they could be inconsistent and misleading [31]. Thus it is important to clarify the reliability of cell nanoparticle toxicity assessment. Whether the excessive subculture could disturb the response of cultured cells to nanoparticles was studied, so as to obtain consistent toxicity test results. The investigators compared the cellular responses to silver nanoparticles across multiple passage numbers in $\mathrm{Ba} / \mathrm{F} 3-\mathrm{BCR}-\mathrm{ABL}$ cells [32]. The action of culture was not found to affect the cell response if the cultured cells were maintained in optimal state. Thus, it is suggested that cells isolated from normal tissues should be applied in the study of nanomaterials exposure on cells.

It is highly promising that nanotechnologies be applied in biomedicine (Table 1). Nanomaterial-based contrast agents in molecular imaging usually have higher sensitivity and possibly fewer side-effects, and can circulate in the blood for longer time. They are also potential prominent drug carriers in the research, diagnosis and therapy of multiple diseases, and are potential cancer drugs targeting on the tumor microenvironment. The unique property of nanomaterials for drug delivery and antineoplastic function highlights the new drug development in the future. Therefore the nanomaterial biosafety needs to be assessed systematically. What's more, nanotechonologies applied in basic biomedical research create a novel platform at the nano-scale to study and develop novel therapies for multiple diseases.

This work was supported by the National Basic Research Program of China (2011CB503903), the National Natural Science Foundation of China (81070078, 81270157) and Beijing Municipal Natural Science Foundation (7102158).

1 Xiao H, Zhang Y. Cutting edge advances in nanomedicine. Sci China Life Sci, 2012, 55: 841-842 
2 Liu C, Qu Y, Luo Y, et al. Recent advances in single-molecule detection on micro- and nano-fluidic devices. Electrophoresis, 2011, 32: 3308-3318

3 Luo W, He K, Xia T, et al. Single-molecule monitoring in living cells by use of fluorescence microscopy. Anal Bioanal Chem, 2013, 405: 43-49

4 He K, Fu Y, Zhang W, et al. Single-molecule imaging revealed enhanced dimerization of transforming growth factor beta type ii receptors in hypertrophic cardiomyocytes. Biochem Biophys Res Commun, 2011, 407: 313-317

5 Liu F, He K, Yang X, et al. Alphala-adrenergic receptor induces activation of extracellular signal-regulated kinase 1/2 through endocytic pathway. PLoS One, 2011, 6: e21520

6 Tong S, Cradick T J, Ma Y, et al. Engineering imaging probes and molecular machines for nanomedicine. Sci China Life Sci, 2012, 55: 843-861

7 Steffens C, Leite F L, Bueno C C, et al. Atomic force microscopy as a tool applied to nano/biosensors. Sensors (Basel), 2012, 12: 82788300

8 Shi X, Qin L, Zhang X, et al. Elasticity of cardiac cells on the polymer substrates with different stiffness: An atomic force microscopy study. Phys Chem Chem Phys, 2011, 13: 7540-7545

9 Dorobantu L S, Goss G G, Burrell R E. Atomic force microscopy: A nanoscopic view of microbial cell surfaces. Micron, 2012, 43: $1312-1322$

10 Mateu M G. Mechanical properties of viruses analyzed by atomic force microscopy: A virological perspective. Virus Res, 2012, 168: 1-22

11 Fotiadis D. Atomic force microscopy for the study of membrane proteins. Curr Opin Biotechnol, 2012, 23: 510-515

12 Nie X, Chen C. Au nanostructures: An emerging prospect in cancer theranostics. Sci China Life Sci, 2012, 55: 872-883

13 Lee H Y, Li Z, Chen K, et al. Pet/mri dual-modality tumor imaging using arginine-glycine-aspartic (rgd)-conjugated radiolabeled iron oxide nanoparticles. J Nucl Med, 2008, 49: 1371-1379

14 Jun Y W, Huh Y M, Choi J S, et al. Nanoscale size effect of magnetic nanocrystals and their utilization for cancer diagnosis via magnetic resonance imaging. J Am Chem Soc, 2005, 127: 5732-5733

15 Lee J H, Huh Y M, Jun Y W, et al. Artificially engineered magnetic nanoparticles for ultra-sensitive molecular imaging. Nat Med, 2007, 13: 95-99

16 Zhang Z, Wang L, Wang J, et al. Mesoporous silica-coated gold nanorods as a light-mediated multifunctional theranostic platform for cancer treatment. Adv Mater, 2012, 24: 1418-1423
17 Chi A H, Clayton K, Burrow T J, et al. Intelligent drug-delivery devices based on micro- and nano-technologies. Ther Deliv, 2013, 4: 77-94

18 Meng E, Hoang T. Micro- and nano-fabricated implantable drug-delivery systems. Ther Deliv, 2012, 3: 1457-1467

19 Gulati K, Aw M S, Findlay D, et al. Local drug delivery to the bone by drug-releasing implants: Perspectives of nano-engineered titania nanotube arrays. Ther Deliv, 2012, 3: 857-873

20 Kennedy L C, Bickford L R, Lewinski N A, et al. A new era for cancer treatment: Gold-nanoparticle-mediated thermal therapies. Small, 2011, 7: 169-183

21 Arora P, Sindhu A, Dilbaghi N, et al. Nano-regenerative medicine towards clinical outcome of stem cell and tissue engineering in humans. J Cell Mol Med, 2012, 16: 1991-2000

22 Verma S, Domb A J, Kumar N. Nanomaterials for regenerative medicine. Nanomedicine (Lond), 2011, 6: 157-181

23 Brammer K S, Frandsen $\mathrm{C}$ J, Jin $\mathrm{S}_{\text {. }} \mathrm{TiO}_{2}$ nanotubes for bone regeneration. Trends Biotechnol, 2012, 30: 315-322

24 Chen C, Xing G, Wang J, et al. Multihydroxylated [GD@ $\left.\mathrm{C}_{82}(\mathrm{OH})_{22}\right]_{n}$ nanoparticles: Antineoplastic activity of high efficiency and low toxicity. Nano Lett, 2005, 5: 2050-2057

25 Dang S, Liu Q, Zhang X, et al. Comparative cytotoxicity study of water-soluble carbon nanoparticles on plant cells. J Nanosci Nanotechnol, 2012, 12: 4478-4484

26 Alkilany A M, Nagaria P K, Hexel C R, et al. Cellular uptake and cytotoxicity of gold nanorods: Molecular origin of cytotoxicity and surface effects. Small, 2009, 5: 701-708

27 Vardharajula S, Ali S Z, Tiwari P M, et al. Functionalized carbon nanotubes: Biomedical applications. Int J Nanomedicine, 2012, 7: 5361-5374

28 Davda J, Labhasetwar V. Characterization of nanoparticle uptake by endothelial cells. Int J Pharm, 2002, 233: 51-59

29 Park J S, Han T H, Lee K Y, et al. N-acetyl histidine-conjugated glycol chitosan self-assembled nanoparticles for intracytoplasmic delivery of drugs: Endocytosis, exocytosis and drug release. J Control Release, 2006, 115: 37-45

30 Kostarelos K, Lacerda L, Pastorin G, et al. Cellular uptake of functionalized carbon nanotubes is independent of functional group and cell type. Nat Nanotechnol, 2007, 2: 108-113

31 Fischer H C, Chan W C. Nanotoxicity: The growing need for in vivo study. Curr Opin Biotechnol, 2007, 18: 565-571

32 Guo D, Zhang X, Huang Z, et al. Comparison of cellular responses across multiple passage numbers in balf3-bcr-abl cells induced by silver nanoparticles. Sci China Life Sci, 2012, 55: 898-905

Open Access This article is distributed under the terms of the Creative Commons Attribution License which permits any use, distribution, and reproduction in any medium, provided the original author(s) and source are credited. 